\title{
Dificuldades vivenciadas pelo enfermeiro assistencial nas unidades de urgência e emergência: uma revisão integrativa
}

\author{
Difficulties experienced by nursing assistants in urgency and emergency units: An integrative \\ review
}

Dificultades experimentadas por los auxiliares de enfermería en Unidades de urgencia y emergencia: Una revisión integradora

\section{Resumo}

$\mathrm{O}$ atendimento às urgências e emergências tem sido alvo de discursão em vários países, em função do seu impacto na atenção à saúde e nos indicadores de morbidade e mortalidade. No Brasil, diversos autores vêm discutindo a atenção prestada às urgências e emergências sob enfoques variados. $O$ objetivo deste estudo se refere a investigar as evidências cientificas acerca das principais dificuldades vivenciadas pelo enfermeiro na assistência dentro das unidades de urgência e emergência no período de 2015 a 2020. Trata-se de uma pesquisa descritiva com abordagem qualitativa acrescida por meio do método de Revisão Integrativa da Literatura (RIL), nas bases de dados LILACS, BDENF E MEDLINE. Para a análise de dados utilizou-se a proposta de Bardin, com o auxílio do programa IRAMUTEC na exploração do material. Obteve-se 3 classes, sendo elas: A importância do enfermeiro no ambiente hospitalar de urgência e emergência; Exposição dos profissionais de enfermagem a fatores de desgaste físicos e psicológicos que afetam a qualidade do atendimento ao paciente; $\mathrm{O}$ desenvolvimento da assistência relacionados aos recursos materiais e infraestrutura nos serviços de urgência e emergência. Foi possível observar as inapropriadas condições de trabalho a que os profissionais de enfermagem estão expostos em seu cotidiano, as quais os colocam em risco à aquisição de doenças infecciosas, exposição à violência e estresse ocupacional, comprometendo a realização de uma assistência qualificada e humanizada.

Palavras-chave: Enfermagem; Urgência; Emergência.

\begin{abstract}
Urgency and emergency care has been the subject of discussion in several countries, due to its impact on health care and on morbidity and mortality indicators. In Brazil, several authors have been discussing the attention paid to urgencies and emergencies under different approaches. The objective of this study is to investigate the scientific evidence about the main difficulties experienced by nurses in care in urgent and emergency units in the period from 2015 to 2020. This is a descriptive research with a qualitative approach added through the Review method Integrativa da Literature (RIL), in the LILACS, BDENF AND MEDLINE. For data analysis, Bardin's proposal was used, with the aid of the IRAMUTEC program in the exploration of the material. 3 classes were obtained, namely: The importance of nurses in the hospital environment of urgency and emergency; Exposure of nursing professionals to physical and psychological stress factors that affect the quality of patient care; The development of assistance related to material resources and infrastructure in urgent and emergency services. It was possible to observe the inappropriate working conditions that nursing professionals are exposed to in their daily lives, which put them at risk for the acquisition of infectious diseases, exposure to violence, occupational stress.
\end{abstract}

Keywords: Nursing; Urgency; Emergency. 


\section{Resumen}

La atención de urgencia y emergencia ha sido tema de discusión en varios países, por su impacto en la atención de salud y en los indicadores de morbilidad y mortalidad. En Brasil, varios autores han discutido la atención prestada a las urgencias y emergencias bajo diferentes enfoques. El objetivo de este estudio es investigar la evidencia científica sobre las principales dificultades experimentadas por los enfermeros en atención dentro de las unidades de urgencia y emergencia en el período de 2015 a 2020. Se trata de una investigación descriptiva con abordaje cualitativo agregado a través del método de Revista Integrativa de Literatura (RIL), en bases de datos LILACS, BDENF y MEDLINE. Para el análisis de los datos se utilizó la propuesta de Bardin, con la ayuda del programa IRAMUTEC en la exploración del material. Se obtuvieron 3 clases, a saber: La importancia de las enfermeras en el ámbito hospitalario de urgencia y emergencia; Exposición de los profesionales de enfermería a factores de estrés físico y psicológico que afectan la calidad de la atención al paciente; El desarrollo de asistencia relacionada con recursos materiales e infraestructura en servicios de urgencia y emergencia. Fue posible observar las condiciones de trabajo inadecuadas a las que se encuentran expuestos los profesionales de enfermería en su vida diaria, que los ponen en riesgo de adquirir enfermedades infecciosas, exposición a la violencia y el estrés laboral, comprometiendo la prestación de asistencia calificada y humanizada.
\end{abstract}

Palabras clave: Enfermería; Urgencia; Emergencia.

\title{
1. Introdução
}

As atividades hospitalares de urgência e emergência possuem características próprias que influenciam a estruturação do trabalho. Um dos ambientes de saúde considerados mais estressantes é a unidade de emergência, pois refere-se ao local que se presta atendimento ao paciente em situação de risco imediato. Assim atribui-se ao termo urgência, a ocorrência repentina de agravo à saúde com ou sem risco possível de morte, cujo usuário necessita de assistência médica em caráter menos imediatista e emergência situações que impliquem em risco iminente de morte ou sofrimento intenso. Diante disso, este setor possui características que produzem estresse em função da especificidade das ações que são desenvolvidas (Oliveira et al., 2019).

Segundo Souza et al., (2019), os serviços de urgência e emergência (SUE) são fundamentais quando se refere a assistência em saúde e classificados como serviços de porta aberta no Sistema Único de Saúde (SUS). Ainda assim, têm-se a sobrecarga de serviços, sob a razão de diversos fatores, pode-se destacar: grande procura, deficiência na questão estrutural das redes de atenção à saúde, insuficiência no dimensionamento de recursos humanos, carência de recursos materiais, violência e acidentes de trânsito, o que compromete a qualidade do atendimento.

O trabalhador de enfermagem está em comunicação direta e constante com o paciente grave e suas complexidades. Este passa a desenvolver um novo papel, como o profissional que irá organizar a assistência, atuando como conservador das normas e rotinas do local de trabalho, que dimensiona o conhecimento e prática, ascendendo à assistência qualitativa (Pissaia, \& Thomas, 2019). Nesse contexto o enfermeiro possui um papel importante e fundamental no qual está relacionado aos cuidados e procedimentos decisivos, e precisam estar aptos a responder por suas ações, deveres, realizar tomada de decisão em tempo hábil para serem implementadas avaliando o paciente de forma eficiente (Lima et al., 2020).

No entanto, o profissional enfermeiro na unidade de urgência sente-se desvalorizado, o seu trabalho é sobrecarregado com acumulação de funções, gerando desgaste físico e emocional causado pelos conflitos operacionais, pelas atividades exercidas com poucos recursos físicos, número reduzido de profissionais, somado ao salário não condizente (Souza, Pessoa Júnior \& Miranda, 2017).

Dessa forma, faz-se necessário que o serviço proporcione condições de trabalho adequadas, dispondo aos profissionais alternativas que os motivem, acarretando em uma maior dedicação na execução de suas atividades, por conseguinte, um melhor atendimento à população (Angelim \& Rocha, 2016).

Perante esta abordagem, o objetivo deste estudo foi investigar as evidências cientificas acerca das principais dificuldades vivenciadas pelo enfermeiro na assistência nos serviços de urgência e emergência no período de 2015 a 2020. 
Como questão norteadora de pesquisa apresenta-se: Quais as principais dificuldades vivenciadas pelo enfermeiro assistencial nas unidades de urgência e emergência descritas nas evidências cientificas no período de 2015 a 2020 ?

\section{Metodologia}

Trata- se de uma pesquisa acrescida por meio do método de Revisão Integrativa da Literatura (RIL) através do modelo descritivo, com abordagem qualitativa. A pesquisa qualitativa, considera o pesquisador como principal instrumento. Seus dados são, de preferência, descritivos, o processo torna-se predominante em relação ao resultado, a significação dada pelos sujeitos participantes são os objetos de atenção do pesquisador e, a análise dos resultados obtidos segue um processo com intuito de chegar a uma conclusão (Pereira et al., 2018). A RIL fundamenta- se em um tipo de pesquisa que examina de forma ampla os estudos científicos tendo como finalidade simplificar as ideias expostas e também preencher as lacunas encontradas com incentivo de novas pesquisas. Deste modo, a revisão integrativa é o modelo de estudo com maior dimensão que permite a inserção de artigos científicos, proporcionando a maior compreensão sobre uma situação problema (Bardin, 2016).

Para a realização da RIL deve-se seguir as etapas: 1) Instituir hipóteses ou questão de investigação; 2) Amostragem ou busca na literatura; 3) Classificação das pesquisas; 4) Análise da literatura incluída na revisão; 5) Interpretação dos desfechos; 6) Síntese do estudo ou apresentação da revisão (Marconi \& Lakatos, 2017).

$\mathrm{Na}$ primeira etapa formulou-se a questão da pesquisa: quais as evidencias cientificas acerca das dificuldades vivenciadas pelo profissional de enfermagem na urgência e emergência no período de 2015 a 2020 ? Na segunda etapa se estabeleceu a amostra como também se definiu as bases de dados e critérios de inclusão e exclusão. Sendo assim a busca foi realizada em consulta a biblioteca virtual da saúde (BVS) nas bases de dados a Literatura científica e técnica da América Latina e Caribe (LILACS), Sistema Online de Busca e Análise de Literatura Médica (MEDLINE) e Base de Dados em Enfermagem (BDENF). Em que foram incluídos artigos completos, originais e de revisão, na língua portuguesa. Excluindo teses, dissertações, relato de experiência, estudo de casos, livros e manuais. Buscou-se com o cruzamento dos seguintes descritores através do operador booleano AND: enfermagem, urgência, emergência.

$\mathrm{Na}$ terceira etapa selecionou-se estudos que se encaixaram nos seguintes critérios de inclusão: artigos científicos publicados no período de 2015 a 2020, de periódicos completos em português, revisões integrativas e sistemáticas, artigos completos e originais. Foram excluídos artigos repetidos, fora do período determinado, artigos em língua estrangeira e que não abordavam a temática sugerida.

Para extrair os dados coletados dos artigos selecionados, fez- se necessário a utilização de um instrumento previamente elaborado e validado capaz de assegurar que a totalidade dos dados relevantes fosse extraída, minimizando os riscos de erros na transcrição, garantindo precisão e checagem das informações e servindo como registro (Ursi \& Galvão, 2005).

Para a análise que corresponde a quarta etapa, utilizou-se o Conteúdo de Bardin que possui três etapas: pré-análise, exploração do material e a análise, cujo objetivo é a apreciação crítica de análises de conteúdo nas pesquisas qualitativas e quantitativas. Utilizados os termos da pesquisa acima descritos e a partir dos resultados filtrados, foram escolhidos os artigos pelos títulos e resumos. Após isso, foram lidos para avaliação completa e verificação de elegibilidade, a fim de determinar o número de artigos para análise qualitativa (Bardin, 2016).

Para auxiliar na análise dos dados será utilizado o software livre IRAMUTEQ (Interface de $R$ pour les Analyses Multidimensionelles de Textes et de Questionnaries), aonde optou-se pelo uso da classificação hierárquica descendente (CHD) para realizar a análise textual da pesquisa. A CHD é composta por três etapas: a preparação e a codificação do texto inicial, a classificação hierárquica descendente realizada pelo processamento de dados e a interpretação das classes. As classes são 
formadas segunda a relação dos vários segmentos de textos (ST) processados e que apresentam palavras homogêneas. Após o processamento e o agrupamento quanto as ocorrências das palavras, a CHD cria o dendograma das classes (Souza et al., 2018).

Na sexta etapa definiu-se os resultados em categorias de acordo com as palavras que mais se repetiram na análise dos copus textuais inseridos no software do IRAMUETQ e assim iniciou-se a discussão de acordo com os estudos de outros autores sobre o tema.

\section{Resultados}

A amostragem inicial desta pesquisa foi de 550 artigos sendo, 266 extraídos da BDENF, 243 extraídos do LILACS e 41 extraídos do MEDLINE. Após a aplicação do primeiro filtro baseado nos critérios de inclusão e exclusão restaram 34 publicações, sendo 16 pertencentes a base da BDENF, 16 na base da LILACS e 2 pertencentes a base do MEDLINE. Após a aplicação do segundo filtro, verificando os estudos com afinidade com a temática desta pesquisa, restou 20 artigos, 4 pertencentes a BDENF, 15 pertencentes a base de dados da LILCAS e 1 estudo pertencente ao MEDLINE. A Figura 1 ilustra o fluxo de seleção dos artigos utilizados.

Figura 1. Fluxograma de seleção e inclusão dos artigos na revisão.

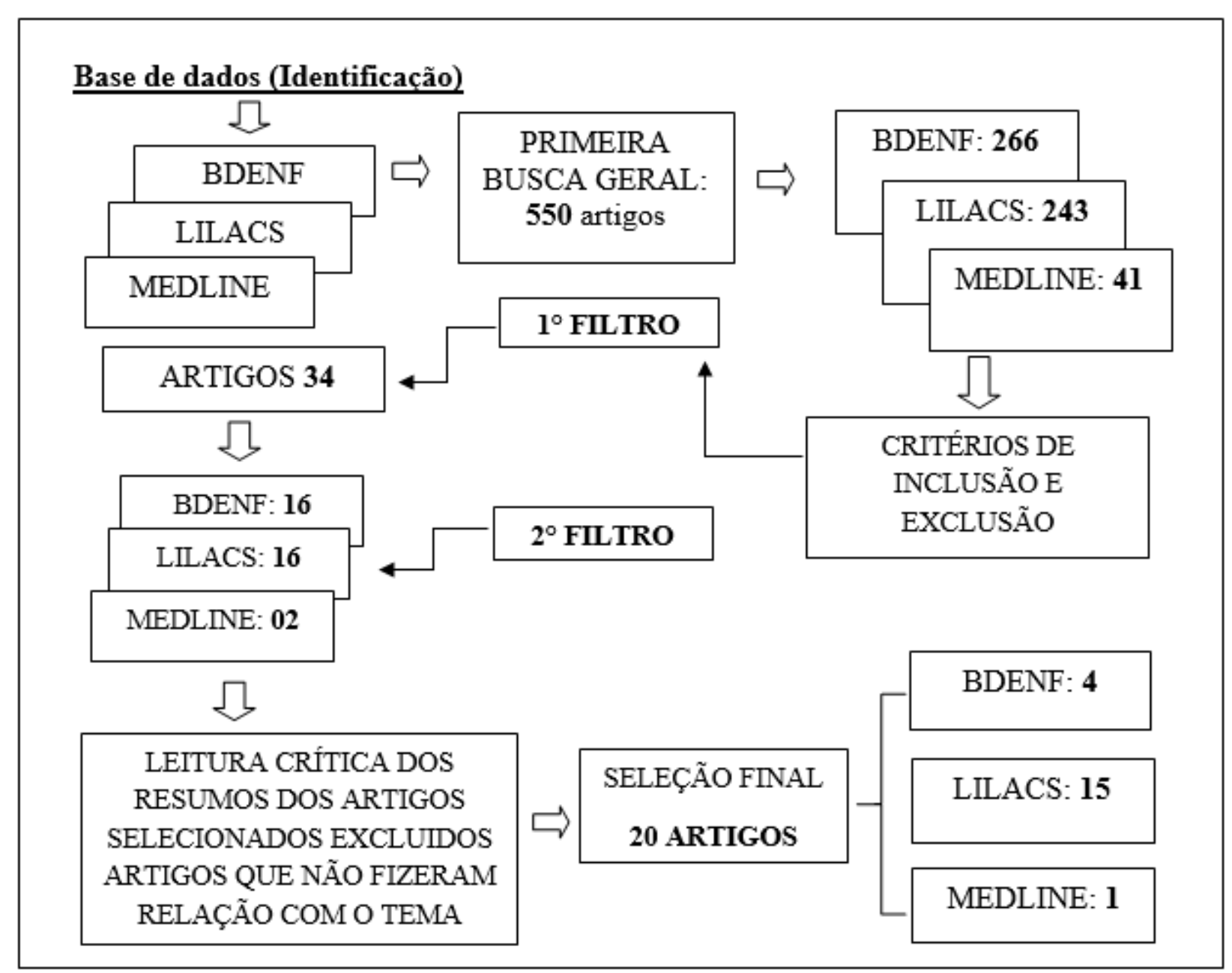

Fonte: Autores (2020). 
Os artigos selecionados foram lidos na íntegra e minuciosamente analisados para compor os dados da amostra da pesquisa, conforme serão apresentados a seguir (Quadro 1).

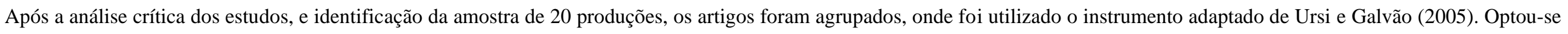
por selecionar os itens: Artigo, autores, base de dados, ano e objetivo.

Quadro 1. Síntese dos artigos selecionados da RIL, 2015-2020.

\begin{tabular}{|c|c|c|c|c|}
\hline Artigo & Autores & $\begin{array}{l}\text { Base de } \\
\text { dados }\end{array}$ & Ano & Objetivo \\
\hline $\begin{array}{l}\text { Satisfação do enfermeiro no serviço de } \\
\text { atendimento móvel de urgência aéreo }\end{array}$ & LIMA, E. W. M; et al. & BDENF & 2015 & $\begin{array}{c}\text { Descrever a satisfação do enfermeiro no Serviço de Atendimento Móvel de } \\
\text { Urgência Aéreo e Identificar os problemas que dificultam a assistência de } \\
\text { enfermagem no Samu Aéreo. }\end{array}$ \\
\hline $\begin{array}{l}\text { Sintomas de depressão e fatores } \\
\text { intervenientes entre enfermeiros de serviço } \\
\text { hospitalar de emergência }\end{array}$ & $\begin{array}{l}\text { OLIVEIRA, F. P; } \\
\text { MAZZAIA, M. C; } \\
\text { MARCOLAN, J. F. }\end{array}$ & LILACS & 2015 & $\begin{array}{c}\text { Averiguar se enfermeiros da área hospitalar de emergência manifestavam } \\
\text { sintomas depressivos; constatar os fatores intervenientes e analisar sobre como } \\
\text { percebem o sofrimento psíquico e como este influencia na assistência } \\
\text { realizada. }\end{array}$ \\
\hline $\begin{array}{l}\text { Equipes e condições de trabalho nos serviços } \\
\text { de atendimento pré-hospitalar móvel: } \\
\text { revisão integrativa }\end{array}$ & PAI, D. D; et al. & LILACS & 2015 & $\begin{array}{l}\text { Identificar, analisar e sintetizar os dados detectados na literatura sobre a } \\
\text { composição das equipes e quais as suas condições de trabalho nos serviços de } \\
\text { Atendimento Pré-Hospitalar (APH) móvel. }\end{array}$ \\
\hline $\begin{array}{l}\text { Risco de adoecimento dos profissionais de } \\
\text { enfermagem no trabalho em atendimento } \\
\text { móvel de urgência }\end{array}$ & WORM, F. A; et al. & LILACS & 2016 & $\begin{array}{c}\text { Mapear os fatores de risco ao adoecimento relacionado ao trabalho dos } \\
\text { profissionais de Enfermagem da Equipe do Serviço de Atendimento Móvel de } \\
\text { Urgência. }\end{array}$ \\
\hline $\begin{array}{l}\text { Produção científica acerca das condições de } \\
\text { trabalho da enfermagem em serviços de } \\
\text { urgência e emergência }\end{array}$ & $\begin{array}{l}\text { ANGELIM, R. C. M; } \\
\text { ROCHA, G.S.A. }\end{array}$ & LILACS & 2016 & $\begin{array}{l}\text { Investigar as produções científicas sobre as condições de trabalho do pessoal } \\
\text { de enfermagem no setor de urgência e emergência. }\end{array}$ \\
\hline
\end{tabular}


Research, Society and Development, v. 10, n. 2, e29510212487, 2021

(CC BY 4.0) | ISSN 2525-3409 | DOI: http://dx.doi.org/10.33448/rsd-v10i2.12487

\begin{tabular}{|c|c|c|c|c|}
\hline $\begin{array}{l}\text { Desvelando situações de risco no contexto } \\
\text { de trabalho da Enfermagem em serviços de } \\
\text { urgência e emergência }\end{array}$ & LORO, M. M; et al. & LILACS & 2016 & $\begin{array}{l}\text { Conhecer a percepção de uma equipe de enfermagem acerca dos riscos } \\
\text { ocupacionais, no contexto do trabalho de enfermagem, em serviços de } \\
\text { urgência e emergência e identificar as medidas de proteção à saúde utilizadas. }\end{array}$ \\
\hline $\begin{array}{l}\text { A violência contra os profissionais da } \\
\text { enfermagem no setor de acolhimento com } \\
\text { classificação de risco }\end{array}$ & FREITAS, R. J. M; et al. & LILACS & 2017 & $\begin{array}{c}\text { Conhecer os tipos de violência e os fatores que contribuem para os atos } \\
\text { violentos sofridos pela equipe de enfermagem no acolhimento com } \\
\text { classificação de risco (ACCR). }\end{array}$ \\
\hline $\begin{array}{l}\text { Prazer e sofrimento de trabalhadores de } \\
\text { enfermagem de um pronto-socorro }\end{array}$ & MIORIN, J. D; et al. & LILACS & 2018 & $\begin{array}{l}\text { Identificar as situações de prazer e de sofrimento presentes no dia-a-dia do } \\
\text { trabalho para a equipe de enfermagem do Pronto Socorro. }\end{array}$ \\
\hline $\begin{array}{l}\text { Entraves no atendimento pré-hospitalar do } \\
\text { Samu: percepção dos enfermeiros }\end{array}$ & MATA, K. S. S; et al. & BDENF & 2018 & $\begin{array}{l}\text { Verificar a existência de dificuldades no atendimento do Samu na percepção } \\
\text { dos enfermeiros. }\end{array}$ \\
\hline $\begin{array}{l}\text { Potencialidades e limites da autonomia do } \\
\text { enfermeiro em ambiente hospitalar }\end{array}$ & $\begin{array}{l}\text { BONFADA, M. S; } \\
\text { PINNO, C; } \\
\text { CAMPONOGARA, S. }\end{array}$ & BDENF & 2018 & $\begin{array}{l}\text { Identificar os fatores que interferem na autonomia profissional do enfermeiro } \\
\text { no ambiente hospitalar. }\end{array}$ \\
\hline $\begin{array}{l}\text { Satisfação profissional de enfermeiros em } \\
\text { uma unidade de emergência }\end{array}$ & CORDEIRO, M. G; et al. & LILACS & 2019 & $\begin{array}{l}\text { Investigar a satisfação profissional de enfermeiros em uma unidade } \\
\text { de emergência de um hospital da Zona Norte do Ceará. }\end{array}$ \\
\hline $\begin{array}{l}\text { Atendimento a pacientes em situação de } \\
\text { urgência: do serviço pré-hospitalar móvel ao } \\
\text { serviço hospitalar de emergência }\end{array}$ & CUNHA, V. P; et al. & LILACS & 2019 & $\begin{array}{l}\text { Compreender o atendimento do paciente em situação de urgência desde o } \\
\text { serviço pré-hospitalar móvel ao serviço hospitalar de emergência. }\end{array}$ \\
\hline $\begin{array}{l}\text { Auto percepção do estresse ocupacional na } \\
\text { equipe de enfermagem de um serviço de } \\
\text { emergência }\end{array}$ & SILVA, P. N; et al. & LILACS & 2019 & $\begin{array}{l}\text { Identificar o perfil e a auto percepção do estresse na equipe de enfermagem } \\
\text { atuante em setor de emergência. }\end{array}$ \\
\hline
\end{tabular}


Research, Society and Development, v. 10, n. 2, e29510212487, 2021

(CC BY 4.0) | ISSN 2525-3409 | DOI: http://dx.doi.org/10.33448/rsd-v10i2.12487

\begin{tabular}{|c|c|c|c|c|}
\hline $\begin{array}{l}\text { Necessidades de conforto de pacientes } \\
\text { atendidos no serviço de urgência e } \\
\text { emergência: implicações para enfermagem }\end{array}$ & PONTE, K. M. A; et al. & LILACS & 2019 & $\begin{array}{l}\text { Descrever conforto para pacientes em serviço de emergência e implicações no } \\
\text { cuidado de enfermagem. }\end{array}$ \\
\hline $\begin{array}{l}\text { O esgotamento físico dos enfermeiros no } \\
\text { setor de urgência e emergência: Revisão } \\
\text { Integrativa }\end{array}$ & $\begin{array}{l}\text { OLIVEIRA, A. P. S; et } \\
\text { al. }\end{array}$ & LILACS & 2019 & $\begin{array}{l}\text { Identificar a presença de riscos de esgotamento ocupacional no desempenho } \\
\text { de suas atividades laborais em uma unidade de urgência e emergência. }\end{array}$ \\
\hline $\begin{array}{l}\text { Estresse ocupacional dos enfermeiros de } \\
\text { urgência e emergência de um hospital } \\
\text { público de Teresina (PI) }\end{array}$ & SANTANA, R. S; et al. & LILACS & 2019 & $\begin{array}{l}\text { Verificar o nível de estresse ocupacional dos enfermeiros pela Escala Bianchi } \\
\text { na unidade de urgência e emergência de um hospital público de Teresina, } \\
\text { Piauí. }\end{array}$ \\
\hline $\begin{array}{l}\text { Estresse Ocupacional: Exposição da Equipe } \\
\text { de Enfermagem de uma Unidade de } \\
\text { Emergência }\end{array}$ & SANTOS, J. N. M; et al. & LILACS & 2019 & $\begin{array}{l}\text { Descrever os fatores estressores para a equipe de enfermagem do setor de } \\
\text { emergência de um hospital público. }\end{array}$ \\
\hline $\begin{array}{l}\text { Humanização nos serviços de urgência e } \\
\text { emergência: contribuições para o cuidado de } \\
\text { enfermagem }\end{array}$ & SOUSA, K. H. J. F; et al. & MEDLINE & 2019 & $\begin{array}{l}\text { Analisar as evidências das pesquisas desenvolvidas sobre a humanização no } \\
\text { atendimento de urgência e emergência, tendo em vista suas contribuiçôes para } \\
\text { o cuidado de enfermagem }\end{array}$ \\
\hline $\begin{array}{l}\text { Características dos enfermeiros no } \\
\text { atendimento pré-hospitalar: concepções } \\
\text { sobre a formação e exercício profissional }\end{array}$ & $\begin{array}{l}\text { ANDRADE, T. F; } \\
\text { SILVA, M. M. J }\end{array}$ & BDENF & 2019 & $\begin{array}{l}\text { Analisar os aspectos dos enfermeiros no Atendimento Pré-Hospitalar, sua } \\
\text { formação profissional e o que dificulta no exercício do seu trabalho. }\end{array}$ \\
\hline $\begin{array}{c}\text { Perfil, dificuldades e particularidades no } \\
\text { trabalho de profissionais dos serviços de } \\
\text { atendimento pré-hospitalar móvel: revisão } \\
\text { integrativa }\end{array}$ & $\begin{array}{l}\text { SOUSA, B. V. N; } \\
\text { TELES, J. F; } \\
\text { OLIVEIRA, E. F. }\end{array}$ & LILACS & 2020 & $\begin{array}{l}\text { Identificar as características do trabalho dos profissionais dos Serviços de } \\
\text { Atendimento Pré-Hospitalar Móvel. }\end{array}$ \\
\hline
\end{tabular}

Fonte: Ursi (2020) adaptado. 
No Quadro 1, foi possível observar que, entre os artigos selecionados e dispostos em ordem cronológica crescente, os principais assuntos abordados estavam relacionados as condições de trabalho da equipe de enfermagem na assistência as urgências e emergências, que corresponde a um problema relevante durante a prática assistencial.

As classes processadas a partir da CHD, representam o sentido do contexto das palavras e a representação do objeto social estudado no trabalho. A CHD desta pesquisa reconheceu 77 segmentos de textos (ST) e reteve 67 destes, $87.01 \%$ do total, ocorrendo a divisão do corpus em 6 classes, do qual por uma relação direta de dependência entre as classes emergiu 3 que compõe este trabalho, sendo representada no Dendograma, figura 2.

Figura 2. Dendograma com a porcentagem de segmentos de textos em cada classe e palavras com maior qui-quadrado $\left(\mathrm{X}^{2}\right)$ fornecido pelo software IRAMUTEQ - 2020.

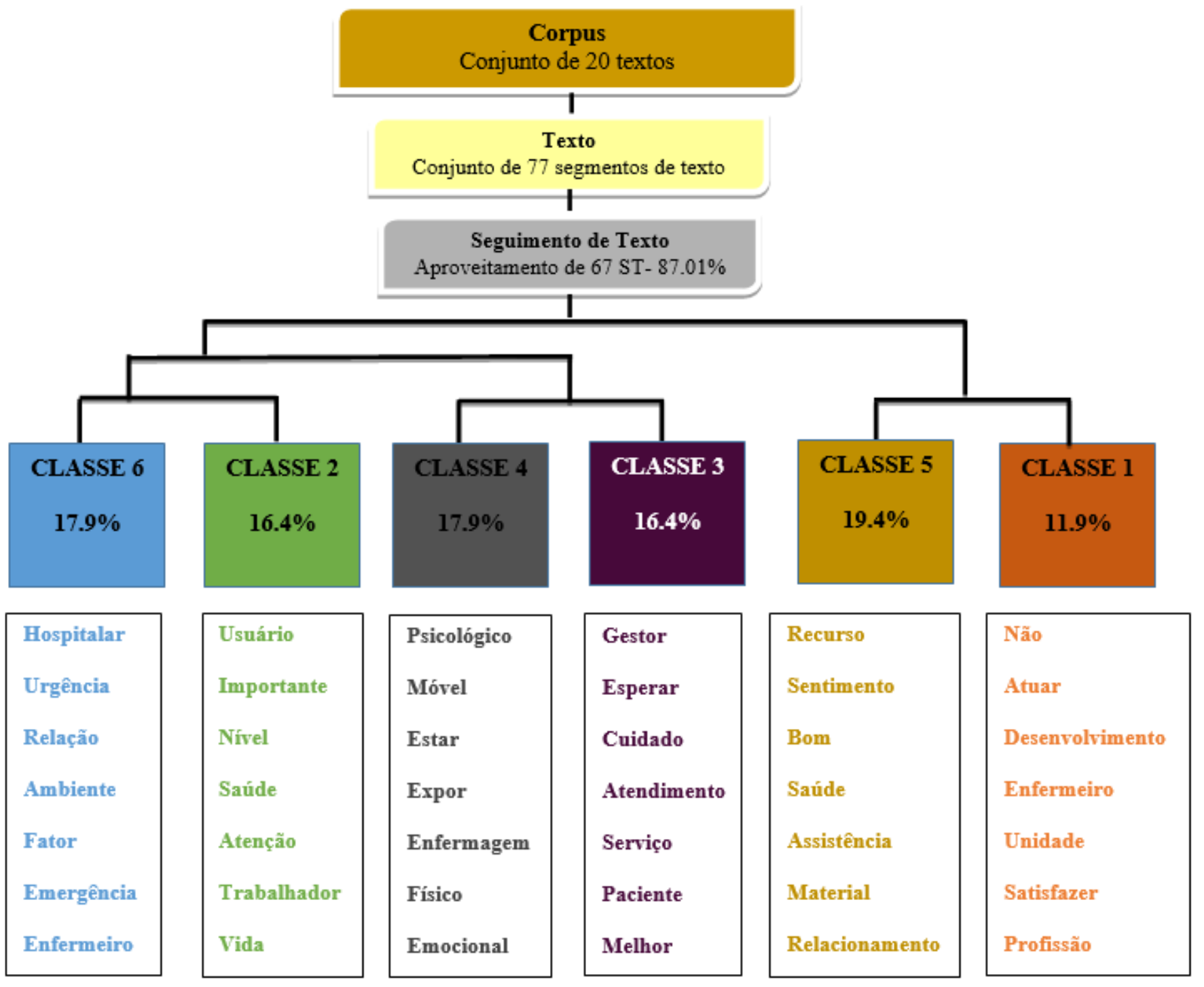

Fonte: Autores.

Por meio do dendograma foi possível visualizar as palavras que obtiveram maior porcentagem quanto à frequência média entre si e diferente entre elas. Esse dicionário de palavras proporcionou, por meio da utilização de qui-quadrado $\left(\mathrm{X}^{2}\right)$, a análise das palavras que apresentaram valor maior que 3,84 e $\mathrm{p}<0,0001$. 
A partir da leitura das palavras em destaque, e de sua inserção nos ST, foi possível alcançar um dos objetivos propostos nesta pesquisa, voltada a verificar nas publicações de artigos científicos as principais dificuldades na assistência à saúde na urgência e emergência, e de como o ambiente e a ausência de estrutura influencia diretamente na qualidade do serviço prestado. As classes apresentaram estreita relação entre si, formando desta maneira pares entre elas.

Classe 6 e 2: De acordo com a classe $6(17.9 \%)$ e a classe $2(16.4 \%)$ que através da análise emergiu, a categoria: A importância do enfermeiro no ambiente hospitalar de urgência e emergência. Podemos destacar que $\mathrm{O}$ profissional enfermeiro tem grande importância no atendimento ao paciente, de forma que o mesmo necessita não somente de habilidade, mas também conhecimento técnico científico e ético na Urgência e Emergência. É um profissional que está diretamente ligado a assistência ao paciente executando tratamentos e coordenando a equipe de enfermagem, responsável por manter a organização, norteamento junto à integração das redes de atenção em todos os seus eixos, além de exercer funções burocráticas. Para isso, deve aliar à fundamentação teórica a capacidade de liderança, o discernimento, a iniciativa, a habilidade de ensinar, a maturidade e a estabilidade emocional.

Classe 4 e 3: A classe 4 (17.9\%) e classe 3 (16.4\%) evidenciaram a exposição do profissional a problemas de cunho psicológico na assistência ao paciente em situação de urgência e emergência, que através da análise emergiu, a categoria: Exposição dos profissionais de enfermagem a fatores de desgaste físicos e psicológicos que afetam a qualidade do atendimento ao paciente. O processo de trabalho no ambiente da urgência e emergência é dinâmico, estimulante e heterogêneo, contudo, pode também expor aos profissionais riscos inerentes ao trabalho na medida em que está cotidianamente responsável pelo cuidado direto ao cliente, exigindo proximidade física, bem como o manuseio constante de equipamentos e materiais onde se faz necessário aprimoramento constante à cerca dos riscos, na perspectiva de diminuir sua exposição, uma vez que, presentes no ambiente laboral, podem determinar a elevação das estimativas de acidente de trabalho e adoecimento do trabalhador.

Classe 5 e 1: Já a classe 5 (19.4\%) e a classe 1 (11.9\%) revelaram a influência dos recursos materiais e de infraestrutura na prestação do serviço à saúde, estando diretamente relacionado a satisfação profissional de atuar no setor de urgência e emergência, que através da análise emergiu, a categoria: $\mathrm{O}$ desenvolvimento da assistência relacionados aos recursos materiais e infraestrutura nos serviços de urgência e emergência. O profissional de enfermagem enfrenta pressões para trabalhar em certas situações relacionado a estrutura do ambiente de trabalho como falta de equipamentos e materiais, superlotação dos serviços, falta de leitos, deficiência no número de funcionários da equipe de enfermagem, acumulo de funções, tarefas repetitivas e monótonas acarretando danos físicos com destaque as "dores nos braços" e "alterações do sono", demonstrando um problema de saúde grave entre os profissionais de enfermagem, os quais podem repercutir na sua qualidade de vida e na capacidade para o trabalho o que implica diretamente na qualidade do atendimento ao usuário que busca o serviço de saúde.

\section{Discussão}

Na primeira categoria, o enfoque identificado foi a importância do enfermeiro no ambiente hospitalar de urgência e emergência. Nesse contexto o enfermeiro possui um papel fundamental, relacionado aos cuidados e procedimentos decisivos, precisam ser capazes de tomada de decisão em tempo hábil avaliando o paciente de forma eficiente (Oliveira et al., 2019).

Desenvolve-se também, ações gerenciais que contribuem para que o atendimento seja prestado com segurança e agilidade, buscando subsídios para a excelência do fazer profissional, participando e coordenando procedimentos que visam à estabilização do quadro clínico do paciente, realizando seu transporte, para que receba um tratamento definitivo, a fim de minimizar possíveis sequelas ao cliente (Mata et al., 2018). 
Os resultados demostraram que a autonomia profissional do enfermeiro está diretamente ligada ao ambiente de trabalho. $\mathrm{O}$ que está relacionado à qualidade assistencial e à satisfação profissional. Nesse sentido, também é possível melhorar o sentimento de valorização e de reconstrução indenitária no momento em que consegue desenvolver seu trabalho com agilidade e segurança (Bonfada, Pinno \& Camponogara, 2018).

Assim, na segunda categoria destaca-se a exposição da equipe de dos profissionais de enfermagem que associaram o sofrimento mental individual ao local em que trabalham, mais especificamente ao pouco suporte ofertado e condições não favoráveis para exercer suas atividades (Oliveira, Mazzaia \& Marcolan, 2015).

A unidade de urgência e emergência é um ambiente onde os profissionais de saúde estão constantemente expostos a riscos, tanto físicos como psíquicos, por se tratar de um serviço em que o atendimento é permeado por pressão, e local cujos os trabalhadores lidam rotineiramente com pacientes em risco iminente de morte (Angelim \& Rocha, 2016).

Este ambiente é apresentado como um importante provocador de situações estressoras, que impactam direta ou indiretamente na qualidade de vida dos trabalhadores e são potenciais desencadeadores de doenças advindas do excesso de carga horária de trabalho, salários não dignos, falta de reconhecimento no âmbito social e grandes responsabilidades com os pacientes. Estas condições irão influenciar de forma significativa na qualidade de vida e saúde do profissional de enfermagem, comprometendo os eixos biopsicossocial destes (Oliveira, Mazzaia \& Marcolan, 2015).

$\mathrm{Na}$ terceira categoria emergiu-se assistência relacionados aos recursos materiais e infraestrutura. Tendo em vista as condições de trabalho deficitárias, diante da falta de recursos materiais e humanos, condições técnicas e falta de atualização, o ambiente de trabalho se torna desumano e, consequentemente, um fator inibitório na efetivação de uma assistência qualificada e humanizada, que busca oferecer um cuidado integral à saúde. Com o objetivo de melhorar as condições básicas de trabalho, vale destacar a necessidade em aumentar o número de trabalhadores, melhorar as estruturas físicas das instituições hospitalares, promover treinamento adequado a todos os profissionais e dispor de recursos materiais necessários (Angelim \& Rocha, 2016).

\section{Conclusão}

Os resultados da pesquisa apontam que, por meio das três categorias apresentadas pelo presente estudo, foi possível observar as inapropriadas condições a qual os profissionais de enfermagem estão submetidos e expostos em seu cotidiano nos serviços de urgência e emergência, as quais colocam em risco esses profissionais à aquisição de doenças infecciosas, exposição à violência, estresse ocupacional, dentre outras abordadas no estudo. Vale ressaltar que, tais agravos à saúde comprometem diretamente na realização de uma assistência qualificada e humanizada.

Além disso, identificou-se que a desvalorização profissional, carga de trabalho extremamente exaustiva e a remuneração financeira baixa, são algumas das razões responsáveis pelo esgotamento físico e mental destes, consentindo que este trabalhador acaba por buscar mais de um vínculo institucional para conseguir manter uma renda mensal digna.

Diante disto o presente estudo possibilitou aprofundar os conhecimentos cientifico, nesse sentido, as dificuldades encontradas pelos enfermeiros na assistência, oferecem subsídios para a reflexão e compreensão dos aspectos intrínsecos na experiência laboral. Por fim, sendo de fundamental importância incentivar a realização desses estudos e de outros que busquem minimizar os riscos e agravos causados à saúde do profissional de enfermagem, bem como elaborar alternativas futuras que visem a melhoria de condições laborais. Recomenda-se a realização de pesquisas futuras com um nível de evidência maior, para avaliação das dificuldades vivenciadas pelo enfermeiro assistencial nas unidades de urgência e emergência, enfatizando a necessidade em aumentar o quantitativo de trabalhadores, a melhoria da estrutura institucional hospitalar, promoção e implementação de treinamento adequado a todos os profissionais e maior disponibilidade de recursos materiais necessários. 


\section{Agradecimentos}

Agradecemos primeiramente a Deus por ter nos dado forças durante esses cinco anos e persistência diante das dificuldades que encontramos no caminho.

Agradecemos a nossa família o amor, carinho, paciência, dedicação, incentivo e ensinamentos que levaremos por toda a vida. Em especial a Gilcelia Oliveira, Luiz Carlos Oliveira e Elza Foro (in memorian) que sempre serão nossa maior inspiração como pessoas.

Agradecemos a todos os nossos amigos que fizeram parte dessa trajetória conosco e que nunca negaram palavras de força, incentivo e otimismo ao longo da nossa jornada acadêmica, (impossível listar um por um), mais sintam-se todos abraçados nesse momento.

Agradecemos a nossa Orientadora Prof ${ }^{a}$ M.Sc. Jorgeany Soares Parente, pela confiança, dedicação, por compartilhar seus conhecimentos, o seu tempo e sua experiência conosco.

Agradecemos a nossa Instituição de ensino UNIFAMAZ, nossa coordenação de enfermagem e todo o corpo docente pela contribuição durante toda a nossa trajetória.

A todos, nossa eterna gratidão!

\section{Referências}

Andrade, T. F. \& Silva, M. J. (2019). Características dos enfermeiros no atendimento pré-hospitalar: Concepções sobre a formação e exercício profissional. Enferm foco, 10(1), 81-86.

Angelim, R. C. M \& Rocha, G. S. A. (2016). Produção científica acerca das condições de trabalho da enfermagem em serviços de urgência e emergência. Revista de pesquisa cuidado é fundamental online, 8(1), 3845-3859.

Bardin, I. (2016). Análise de conteúdo. 229.

Bonfada, M. S., Pinno, C. \& Camponogara, S. (2018). Potencialidades e limites da autonomia do enfermeiro em ambiente hospitalar. Rev enferm UFPE online, 12(8), 2235-2246.

Camargo, B. V \& Justo, A. M. IRAMUTEQ: um software gratuito para análise de dados textuais. Temas Psicol. http://pepsic.bvsalud.org/pdf/tp/v21n2/v21n2a16.pdf.

Cassettari, S. S. R \& Mello, A. L. S. F. (2017) Demanda e tipo de atendimento realizado em unidades de pronto atendimento do município de Florianópolis, Brasil. Texto Contexto Enferm, 26(1), 1-9.

Cordeiro, M. G., et al. (2019). Satisfação profissional de enfermeiros em uma unidade de emergência. Rev nursing, 22(249), $2604-2609$.

Cunha, V. P., et al. (2019). Atendimento a pacientes em situação de urgência: do serviço pré-hospitalar móvel ao serviço hospitalar de emergência. Rev enfermería actual de Costa Rica, 37, 1-15.

Freitas, R. J. M., et al. (2017). A violência contra os profissionais da enfermagem no setor de acolhimento com classificação de risco. Rev gaúcha enferm, $38(3), 1-8$.

Lima, E. W. M., et al. (2015). Satisfação do enfermeiro no serviço de atendimento móvel de emergência aéreo. Rev enferm UFPI, 4(4), 49-55.

Lima, T. F. S., et al. (2020). Dificuldades na coleta de gasometria arterial de pacientes obesos em uma unidade de pronto atendimento: relato de experiência. In: Jorge, W. J. (Ed), Ciências da Saúde - Teoria e Prática (pp. 563-576). Uniedusul.

Loro, M. M., et al. (2016). Desvelando situações de risco no contexto de trabalho da enfermagem em serviços de urgência e emergência. Escola Anna Nery, 20(4), 1-8.

Mata, K. S. S., et al. (2018). Entraves no atendimento pré-hospitalar do samu: percepção dos enfermeiros. Rev enferm UFPE online, 12 (8), $2137-2145$.

Marconi, M. A. \& Lakatos, E. M. (2017). Fundamentos de metodologia científica. (8a ed.) editora atlas.

Miorin, J. D., et al. (2018). Prazer e sofrimento de trabalhadores de enfermagem de um pronto-socorro. Texto contexto enferm, 27(2), 1-9.

Oliveira, A. P. S., et al. (2019). O esgotamento físico dos enfermeiros no setor de urgência e emergência: Revisão integrativa. Revista nursing, 22(251), 28392843 .

Oliveira, F. P., Mazzaia, M. C. \& Marcolan, J. F. (2015). Sintomas de depressão e fatores intervenientes entre enfermeiros de serviço hospitalar de emergência. Acta paul enferm, 28(3) 209-215. 
Research, Society and Development, v. 10, n. 2, e29510212487, 2021 (CC BY 4.0) | ISSN 2525-3409 | DOI: http://dx.doi.org/10.33448/rsd-v10i2.12487

Pai, D. D., et al. (2015). Equipes e condições de trabalho nos serviços de atendimento pré-hospitalar móvel: Revisão integrativa. Rev eletr enferm, 17(4).

Pereira, A. S., et al. (2018). Metodologia da pesquisa científica. UFSM. https://repositorio.ufsm.br/bitstream/handle/1/15824/Lic_C omputacao_MetodologiaPesquisa-Cientifica.pdf?sequence $=1$.

Pissaia, L. F. \& Thomas, J. (2019). Novos cenários da urgência e emergência: intersecções com o $1^{\circ}$ Encontro da Enfermagem Cuidando nas Urgências e Emergências do Coren-RS. Research, Society and Development, 8(5), e685938.

Ponte, K. M. A., et al. (2019). Necessidades de conforto de pacientes atendidos no serviço de urgência e emergência: Implicações para enfermagem. Rer. Fundam. care online, 11(4), 925-930.

Santana, R. S., et al. (2019). Estresse ocupacional dos enfermeiros de urgência e emergência de um hospital público de Teresina (PI). Rev bras med trab, 17(1), 2604-2609.

Santos, J. N. M.O., et al. (2019). Estresse ocupacional: Exposição da equipe de enfermagem de uma unidade de emergência. Rev fundam care online, 11, 455463.

Silva, P. N., et al. (2019). Autopercepção do estresse ocupacional na equipe de enfermagem de um serviço de emergência. Journal health NPEPS, 4(2), 357369.

Souza, B. V. N., et al. (2020). Perfil, dificuldades e particularidades no trabalho de profissionais dos serviços de atendimento pré-hospitalar móvel: Revisão integrativa. Revista enfermería actual, (38), 1-16.

Souza, J. D., Pessoa, J. M. J. \& Miranda, F. A. N. (2017). Estresse em serviço de urgência e os desafios para enfermeiros brasileiros e portugueses. Revista de Enfermagem Referência, 4(12), 107-116.

Souza, K. H. J. F., et al. (2019). Humanização nos serviços de urgência e emergência: contribuições para o cuidado de enfermagem. Rev gaúcha enferm, 40, 1 10. e. 20180263

Souza, M. A., et al. (2018). O uso do software IRAMUTEQ na análise de dados em pesquisas qualitativas. Rev. esc. enferm. USP, 52. e03353.

Ursi, E. S. \& Galvão, C. M. (2006). Prevenção de lesões de pele no perioperatorio: revisão integrativa da literatura. Rev. Latina-Am. Enfermagem, 14(1), 124131.

Worm, F. A., et al. (2016). Risco de adoecimento dos profissionais de enfermagem no trabalho em atendimento móvel de urgência. Rev cuid, 7(2), 1288-1296. 